\title{
Wakeup Call: Reviewing the Effects of Sleep on Decision-Making in Athletes and Implications for Sports Officials
}

\author{
Michele Lastella', Zozan Onay ${ }^{2}$, Aaron T. Scanlan ${ }^{3}$, Nathan Elsworthy ${ }^{4}$, Nathan W. Pitchford ${ }^{5}$, \\ Grace E. Vincent ${ }^{1}$
}

\begin{abstract}
Affiliations: 'Central Queensland University, Appleton Institute for Behavioural Science, Adelaide, SA, Australia ${ }^{2}$ Central Queensland University, School of Health, Medical and Applied Sciences, Brisbane, QLD, Australia, ${ }^{3}$ Central Queensland University, Human Exercise and Training Laboratory, Rockhampton, QLD, Australia, ${ }^{4}$ Central Queensland University, School of Health, Medical and Applied Sciences, Mackay, QLD, Australia, ${ }^{5}$ University of Tasmania, Sports Performance Optimisation Research Team, School of Health Sciences, Launceston, TAS Australia
\end{abstract}

Correspondence: M. Lastella, Central Queensland University, Appleton Institute for Behavioural Science, 44 Greenhill Road, Wayville, SA, 5034, Australia. Email: m.lastella@cqu.edu.au

ABSTRACT Although sports officials are essential in the adjudication of rules during competitions, research into officiating populations is preliminary. This review explores existing research on the effects of sleep loss on decision-making in athlete populations, to gain relevant insight into sports officials and the growing decision-making demands of their role. Specifically, cognitive factors that influence decision-making are identified, and the potential relationship between sleep/wake behaviours and decision-making in sports officials were examined. Particular focus was granted to sleep, specifically sleep restriction in athlete populations, and the hypothesized relationship between sleep/wake behaviours and decision-making in sports officials. Impaired sleep duration and quality has been shown to impair cognitive performance. In the context of sport, impairments to sleep can negatively impact motivation, physical strength, reaction time, and decision-making, all of which are imperative to the role of the sports official. Despite numerous studies demonstrating that sleep loss reduces cognitive performance in athletes, this review revealed that similar research in sports officials has surprisingly yet to be conducted. Since sports officials require a high level of cognitive and physical skills to make correct decisions, an inquiry into the influence of sleep on cognitive performance may encourage research that facilitates improved performance training methods for decision-making in officials. Such findings are important to maintain the standard of officiating and allow for the development of guidelines and strategies to manage sleep and optimize decision-making across various sporting codes.

KEY WORDS referee, umpire, mood, motivation, competition

$@$ MJSSMontenegro

EFFECTS OF SLEEP ON DECISION-MAKING IN SPORT

http://mjssm.me/?sekcija=article\&artid=202

\section{Introduction}

As the professional and recreational market of sport is under more pressure than ever, the need to enforce rules and regulation in sporting events has paralleled the lucrative shift in athletic competition (Stewart \& Smith, 2000). Such regulation is heavily controlled by the sports official. The sports official is fundamental to the regulation of competition in sport, by administering the rules, which influence overall match results based on their adjudication of play and decision-making. Furthermore, officials carry an ethical responsibility that preserves the intrinsic value of sport and competition, all under the watchful eye of athletes, coaches, spectators, and the entire audience of a broadcasted competition (Page \& Page, 2010; Isidori, Muller, \& Kaya, 2012).

Despite the crucial role of sports officials, they have not been extensively considered in research. While athletes often physically benefit from interchanges during competition, audiences seemingly discount the fact that sports officials are required to complete an entire match, limiting their opportunity to recover between

Received: February 062020 | Accepted after revision: March 292020 | First published online: Septembar 012020

(c) 2020 by the author(s). License MSA, Podgorica, Montenegro. This article is an open access article distributed under the terms and conditions of the Creative Commons Attribution (CC BY).

Conflict of interest: None declared. 
bouts of high-intensity activity (Heinemann, 1990; MacMahon et al., 2014; Pina, Passo, \& Maynard, 2018). Sports officials have a range of demands to consider during competition, their role in evaluating technical code, decision-making, and enduring physical demands are consistently present across sports (MacMahon, Helsen, Starkes, \& Weston, 2007; MacMahon et al., 2014).

Since the adjudication of rules is imperative to the role of sports officials, this review will examine their decision-making, including the underlying cognitive factors involved. It is important to note, due to the limited research on this topic, this review will consider key findings reported in athlete populations where relevant. Furthermore, given the well-established importance of sleep to cognitive performance (Belenky et al., 2003; Rogers, Dorrian, \& Dinges, 2003), particular focus will be given to sleep in this review. Specifically, sleep restriction in athlete populations and the potential relationship between sleep/wake behaviours and decision-making in sports officials will be discussed.

This narrative review searched for sleep and decision-making research conducted in both athletes and sports officials. Although narrative, the source articles were identified using a systematic search strategy. The online databases Cochrane, MEDLINE (Pubmed), Scopus and Web of Science for scientific papers were searched using keywords, including "sleep" and "decision" in combination with "athlete", "sport", and "sports official". Relevant references were inspected and limited to peer-reviewed papers published in English from 1981 to 2020.

\section{Decision-making in sports officials}

Decision-making in sport requires sports officials to possess a high level of rule knowledge and application (MacMahon et al., 2007). MacMahon and Plessner (2013) categorized sports officials into three main types; interactors, reactors, and monitors, based on their interaction with athletes and the number of signals being monitored. Interactors enforce competition rules, such as soccer officials, reactors play a perceptual role in monitoring specific competition codes, such as tennis line judges, and monitors observe and assess the quality of performance, for example, gymnastic judges (MacMahon \& Plessner, 2013). Despite these variations in duty across sports, officials all share a common process - decision-making - defined as the ability to integrate information to plan, judge, and execute an action (Causer \& Ford, 2014; MacMahon \& Plessner, 2013).

On occasions, the decision-making requirements of sports officials may be relatively simple. However, it is common for sports officials to undertake complex decision-making processes, requiring a high level of rule interpretation. Recent studies have examined the extent of decision-making during competition, with 887 decisions made by officials during an Australian Football League match (Neville, Salmon \& Read, 2007), and approximately 140 observable decisions made by officials during an international soccer match (Helsen \& Bultynck, 2004). In the latter study, Helsen and Bultynck (2004) showed the number of decisions made by soccer officials was uniformly distributed throughout competition, asserting the need for officials to exercise complete focus for for an entire match (Helsen \& Bultynck, 2004). While this study provides a preliminary investigation into the decision-making demands of sports officials, it forms a very limited body of research from which to draw definitive conclusions. These decisions, made under highly stressful and time-sensitive circumstances, can have a large impact on overall match outcome, athlete safety, spectatorship, and sponsorship (Page \& Page, 2010). Recently, the Federation Internationale de Football Association (2019) introduced the Video Assistant Referee (VAR) to help referees in reviewing decisions made by the head referee. However, this approach has been met with some controversy. For example, Nevill et al. (2002) found that referees who viewed videotaped fouls with crowd noise called significantly fewer fouls for the away team than those presented only with video. It is evident that accurate effectiveness of the VAR is still in its infancy, and further research is required to examine its efficacy (Carlos et al., 2019). Further research is also needed to understand the relationship between sleep and decision-making in the VAR context. Regardless, sports officials, whether video assistants or head officials, are vital members of their sports and are recognized as performers in their own right (Morris \& O'Connor, 2017).

While the demands of the sports official role continue to grow, the pressure of officials to reduce decision-making errors parallels such growth (Dickson, 2002). Studies have reported soccer officials attain $86 \%$ accuracy in decision-making during competition (Mallo, Frutos, Juarez, \& Navarro, 2012), whereas Australian football umpires reported $84 \%$ accuracy when awarding free kicks during competition (Elsworthy, Burke, Scott, Stevens, \& Dascombe, 2014). Regardless of this need for high-quality officiating and decision-making, recent studies have focused on the physical and cognitive factors underpinning decision-making processes in athletes across sports (Almonroeder, Tighe, Miller, \& Lanning, 2018; Konishi et al., 2017; Scanlan, Humphries, Tucker, \& Dalbo, 2014). Because the cognitive skills of athletes and sports officials are specific to their roles, the prevailing research on athletes should not be considered completely transferable to sports officials (Williams \& Davids, 1995).

Physical, environmental, and psychological demands have been recognized as defining factors for decision-making performance in sports officials (Larkin, Mesagno, Berry, \& Spittle, 2018). To understand officiating performance, Mascarenhas et al. (2005) developed a model identifying five common themes: (i) knowledge and application of competition code; (ii) physical fitness; (iii) personality and match management; (iv) contextual judgement; and (v) psychological characteristics. While these themes provide a framework to identify factors that contribute to performance and are theoretically relevant to decision-making ability, they do not describe how such contextual factors might affect the underlying cognitions of decision-making or 
inform and improve the process. Understanding the factors that improve decision-making and the integration of information to enforce competition rules is vital for providing a high standard of decision-making in officials (Helsen \& Bultynck, 2004; Williams \& Davids, 1995).

While some studies have examined the impact of contextual factors such as match-bias or rule changes on decision-making, cognitive factors that also influence decision-making in officials have been less explored (Plessner \& Haar, 2006). In general adult populations, an increased interest in the role of mood, stress, fatigue, and sleep on decision-making ability has emerged (Loewenstein \& Lerner, 2003). In a review by Loewenstein and Lerner (2003), cognitive factors significantly impacted decision-making, with incidental and unexpected emotions and stress affecting decision-making quality. These underlying cognitive factors of decision-making are recognized as significant indicators of an individual's ability to respond to a situation, not only because of the positive effect experienced by the individual, but also their ability to utilize such mechanisms to deal with and influence decision-making (Loewenstein \& Lerner, 2003; Xing \& Sun 2013).

With sports officials, early investigations focused on the influence of common sources of stress on physical and cognitive performance (Taylor, Daniel, Leith \& Burke, 1990; Rainey \& Hardy, 1999; MacMahon et al., 2014; Pina, Passo, \& Maynard, 2018). Taylor et al. (1990) found that stressors, such as a lack of appreciation from spectators, athletes, and coaches, contributed to burnout and high turnover rates in soccer officials. Furthermore, younger sports officials reported greater burnout than older sports officials, which was attributed to older sports officials possessing more effective coping mechanisms. Such findings were later supported by Rainey and Hardy (1999), who reported stress-reducing strategies, such as relaxation or self-regulation techniques, reduced burnout and minimized the decline of cognitive and physical performance in rugby union officials. While interpersonal conflicts and the fear of failure are considered the most stressful influences on sports officials, these factors demonstrated small to moderate relationships with stress and burnout rates (Rainey \& Hardy, 1999; Taylor et al., 1990). In fact, studies indicate sports officials experience changing and differing amounts of stress, and the effect of stress on officiating performance is speculative using evidence provided in generic populations (Mascarenhas et al., 2005; Rainey \& Winterich, 1995; Stewart \& Ellery, 1996).

Suggested cognitive factors that influence decision-making in sports officials include prior decisions, fatigue due to travel, and athlete attitudes (Mascarenhas et al., 2005). While these external influences provide insight into broader psychological phenomena, they do not provide direct evidence on whether psychological factors facilitate decision-making in officials. Research has produced conclusions applicable to general populations or athletes on this matter (Akerstedt, 2007; Almonroeder et al., 2018; Durmer \& Dinges, 2005) however, future research is required to attribute such conclusions to sports officials. In turn, a cognitive factor that is routinely demonstrated as having a significant impact on decision-making abilities in the general population is sleep (Akerstedt, 2007; Almonroeder et al., 2018; Weston et al., 2012).

\section{Sleep and decision-making}

Sleep, defined as a homeostatic behavioural state of reduced movement and sensory reaction that repeatedly reoccurs at 24-hour intervals, is widely regarded as critical to cognitive and physiological function (Gordijn \& Beersma, 2007). The effects of sleep on human cognition and motor functioning are well recognized (Daviaux, Mignardot, Cornu, \& Deschamps, 2014; Frey, Badia, \& Wright Jr, 2004; Rogers et al., 2003). Sleep has many psychological and physiological functions, with a recommended duration of seven to nine hours per day in adults (Hirshkowitz et al., 2015; Watson et al., 2015). Studies have shown mood, fatigue, and cognitive function are routinely impaired by sleep quality, partial sleep restriction, or total sleep deprivation in general populations (Akerstedt, 2007; Frey et al., 2004; Rogers et al., 2003).

Sleep quality, a subjective rating of the efficiency and maintenance of sleep, has been shown to influence physical and cognitive function in general populations (Nebes, Buysse, Halligan, Houck, \& Monk, 2009) and athlete populations (Brandt, Bevilacqua, \& Andrande, 2017; Peppard, Barnet, Rasmuson, Bliwise, \& Hagen, 2017). For instance, Peppard et al. (2017) reported significant positive correlations between sleep quality and cognition, specifically memory, executive function, and attention in the general population. However, this study did not account for common conditions that impair sleep and cognition, such as illness, medication, or psychological disorders (Nebes et al., 2009). In this regard, Nebes et al. (2009) analysed the relationship between cognitive performance and the duration of sleep in older adults from the general population while controlling for confounding conditions. Significant positive correlations were reported between sleep quality and cognitive performance, with no significant relationship observed between sleep duration and cognitive performance (Nebes et al., 2009). Consequently, the results of this study highlight the specific need for future investigations to consider the impact of sleep quality on cognitive function.

Reduced sleep duration impacts specific domains of cognitive performance, including increased memory lapses, cognitive slowing, memory impairment, and decreased vigilance, attention, response time, and capability (Chua et al., 2014). Scott, McNaughton, and Polman (2006) examined the effect of 30-hour sleep deprivation on cognitive and psychomotor function compared to normal sleep in a general adult population. Significant disturbances in mood, reaction time, vigour, and mental fatigue were apparent following sleep deprivation. Although studies tend to focus on the effects of sleep deprivation on cognitive performance, sleep restriction is a more common experience in general adult populations (Demos et al., 2016). Indeed, Demos et al. (2016) showed that following restricted sleep, decision-making remained unaffected, yet partici- 
pants were $30 \%$ more likely to make errors in cognitive ability tasks. Consequently, sleep restriction, like sleep deprivation, impaired reaction time and impulsive actions. This collective evidence suggests that reductions in sleep diminish reaction time, alertness, and cognitive performance (Demos et al., 2016; Scott et al., 2006).

Given that sleep restriction is common in athlete populations, examining the implications of this pattern on cognitive performance is paramount (Blumert et al., 2007; Jarraya et al., 2013; Skein et al., 2013). Restrictions to sleep can negatively impact cognitive performance, reaction time, and decision-making - all of which are essential to the role of athletes (Hirshkowitz et al., 2015; Jarraya et al., 2013; Reilly \& Edwards, 2007; Scott, McNaughton, \& Polman, 2006). Blumert et al. (2007) investigated the effect of 24 hours of sleep deprivation on physical performance and mood in national college weightlifters. Sleep deprivation had no significant influence on physical performance but substantially affected mood state. Similarly, Skein et al. (2013) showed 24 hours of sleep deprivation significantly deteriorated reaction time and accuracy during a word colour recognition test due to reduced alertness and attention in rugby league athletes following competition. While these studies show the impact of sleep deprivation on cognitive performance, the simulated overnight sleep deprivation adopted within the respective methodologies limit the representation of common sleep disruptions encountered by athletes (Blumert et al., 2007; Skein et al., 2013). In this regard, Jarraya et al. (2013) examined the effect of sleep restriction on cognitive performance in handball goalkeepers. Athletes were monitored morning and night following regular sleep and restricted sleep, after which they completed three cognitive tasks. Sleep restriction resulted in significantly poorer reaction time, selective attention, and constant attention, with greater changes observed at night compared to morning demonstrating an additive effect of timing on performance.

Given the majority of research investigating the impact of sleep on cognitive performance has utilized simulated laboratory environments, it is necessary to examine sleep and cognition in a real-world competitive context (Blumert et al., 2007; Jarraya et al., 2013). In doing so, findings on sleep and cognitive performance will carry stronger ecological validity. Further studies should consider utilizing cognitive measures that consider sporting environments to closely parallel the context of competition to establish more definitive conclusions.

\section{Sleep restriction in sport}

In sport, sleep restriction provides realistic insights into the experiences of athletes and sports officials. Sleep restriction can negatively impact physical strength and performance, mood, motivation, reaction time, and decision-making in athletes (Lastella, Lovell, \& Sargent, 2014; Martin, 1981; Samuels, 2012; Thun, Bjorvatn, Flo, Harris, \& Pallesen, 2015; Reilly \& Edwards, 2007). These findings are highly relevant to sporting performance as factors such as stress, travel for matches, nervousness prior to competition, and sleeping in unfamiliar environments can significantly curb sleep duration and quality (Pallesen et al., 2017). A crucial element of training and recovery in athletes is sleep management, as disturbances to sleep potentially compromise athletic performance, cognitive function, mood, and injury risk (Durmer \& Dinges, 2005; Erlacher, Ehrlenspiel, Adegbesan, \& El-Din, 2011).

Unavoidable circumstances can disturb sleep in athletes. Eagles et al. (2016) explored the effect of competition on the duration and efficiency of sleep in professional rugby union athletes. Significant reductions in sleep duration on nights following competition were observed, particularly when matches were played in the evening (Eagles et al., 2016). While sleep was negatively impacted following competition, findings were limited by the lack of definition for "non-game nights" (i.e., nights before a competition or nights before no competition). Furthermore, home matches were exclusively examined, and since it is common for competitions to be held at away venues, representation of a competitive season was somewhat limited (Eagles et al., 2016).

Similarly, Juliff et al. (2015) reported a significant reduction in sleep duration on nights before a competition in athletes from individual and team sports, with thoughts about competition and nervousness identified as the main mechanisms interfering with sleep. The investigated athletes also reported being unaware of strategies to overcome poor sleep, possibly highlighting the need for further monitoring and education of sleep in athlete populations (Juliff et al., 2015). While these studies collectively demonstrate that sleep in sporting environments is dependent on the time of competition (day versus night), and sampled night (habitual versus before competition versus after competition), there is yet to be a broad analysis of how all these factors interact and impact sleep in athletes and sports officials (Eagles et al., 2016; Juliff et al., 2015). A comprehensive investigation into the impact of time of competition on sleep outcomes in sports officials should be considered in future studies.

\section{The need to examine sleep and decision-making in sports officials}

Data examining decision-making in sports officials have attributed performance to contextual factors such as time of competition, regulations, and athlete attitudes (Helsen \& Bultynck, 2004; Larkin et al., 2018). Sports officials need to have exceptional cognitive skills to make correct decisions; thus, inquiry to identify factors that influence decision-making, such as sleep, is necessary and long overdue in this population. Future investigation of sleep restriction on cognitive performance in sports officials will not only extend knowledge but allow for improved strategies to enhance decision-making ability and overall sport competition (Williams \& Davids, 1995). 
Cognitive distress, anxiety, and common mental health conditions in athlete populations have gained increased attention in research (Gouttebarge, Backx, Aoki, \& Kerkhoffs, 2015; Junge \& Feddermann-Demont, 2016), while empirical evidence about the psychology of sports officials remains limited (Gouttebarge, Johnson, Rochcongar, Rosier, \& Kerkhoffs, 2017). The lack of research on officials is surprising, considering they perform in difficult psychosocial environments involving stressors such as physical energy, social pressure, and spectator scrutiny. It is common for sports officials across all playing levels to hold dual occupations, thus exacerbating pressures beyond the context of competition. In a study comparing common mental health concerns of athletes and sports officials, officials were found to have a high risk of mental health concerns similar to athletes (Gouttebarge et al., 2017). While this study was specific to soccer officials and therefore may not be generalizable to other sports, the results suggested specific training and support measures for sports officials should be introduced. Consequently, the authors concluded that role-specific management and training protocols were necessary to improve the psychological performance and well-being within and beyond the context of competition in sports officials (Gouttebarge et al., 2017).

Sleep has been consistently recognized to influence decision-making (Demos et al., 2016; Nebes et al., 2009; Scott et al., 2006), and while athlete populations have been identified as experiencing poor sleep duration and quality compared to general populations (Blumert et al., 2007; Jarraya et al., 2013; Skein et al., 2013), the underlying relationship between sleep and decision-making in sports officials has yet to be examined. Not only is such future research important to maintain the integrity of sport performance, but it may provide an opportunity to improve the standard of officiating in sport (Hancock, Rix-Lievre, \& Cote, 2015; Catteuw, Helsen, Gilis, \& Wagemans, 2009). This knowledge will also allow for the development of guidelines and strategies to help manage sleep and optimize decision-making in officials across various sporting codes.

\section{References}

Akerstedt, T. (2007). Altered sleep/wake patterns and mental performance. Physiology and Behaviour, 90, 209218. doi: 10.1016/j.physbeh.2006.09.007

Almonroeder, T., Tighe, S., Miller, T., \& Lanning, C. (2020). The influence of fatigue on decision-making in athletes: A systematic review. Sports Biomechanics, 19(1), 76-89. doi: 10.1080/14763141.2018.1472798

Belenky, G., Wesensten, N., Thorne, D., Thomas, M., Sing, H., Redmond, D., Russo, M., \& Balkin, T. (2003). Patterns of performance degradation and restoration during sleep restriction and subsequent recovery: A sleep dose-response study. Journal of Sleep Research, 12(1), 1-12. doi: 10.1046/j.1365-2869.2003.00337.x

Blumert, P., Crum, A., Ernsting, M., Volek, J., Hollander, D., Haff, E., \& Haff, G. (2007). The acute effects of twenty-four hours of sleep loss on the performance of national-caliber male collegiate weightlifters. Journal of Strength and Conditioning Research, 21(4), 1146-1154. doi: 10.1519/R-21606.1

Brandt, R., Bevilacqua, G., \& Andrande, A. (2017). Perceived sleep quality, mood states, and their relationship with performance among Brazilian elite athletes during a competitive period. Journal of Strength and Conditioning Research, 31(4), 1033-1039. doi: 10.1519/JSC.000000000001551

Carlos L-P., Ezequiel R., \& Anton, K. (2019). How does Video Assistant Referee (VAR) modify the game in elite soccer? International Journal of Performance Analysis in Sport, 19(4), 646-653. doi: $10.1080 / 24748668.2019 .1646521$

Catteuw, P., Helsen, W., Gilis, B., \& Wagemans, J. (2009). Decision-making skills, role specificity, and deliberate practice in association football refereeing. Journal of Sports Sciences, 27(11), 1125-1136. doi: $10.1080 / 02640410903079179$

Causer, J., \& Ford, P. (2014). "Decisions, decisions, decisions": Transfer and specificity of decision-making skill between sports. Cognitive Process, 15(3), 385-389. doi: 10.1007/s10339-014-0598-0

Chua, E., Yeo, S., Lee, I., Tan, L., Lau, P., Cai, S., Zhang, X., Puvanendran, K., \& Gooley, J. (2014). Sustained attention performance during sleep deprivation associates with instability in behavior and physiologic measures at baseline. Sleep Research Society, 37(1), 27-39. doi: 10.5665/sleep.3302

Daviaux, Y., Mignardot, J., Cornu, C., \& Deschamps, T. (2014). Effects of total sleep deprivation on the perception of action capabilities. Experimental Brain Research, 232(7), 2243-2253. doi: 10.1007/s00221014-3915-Z

Demos, K., Hart, C., Sweet, L., Mailloux, K., Trautvetter, J., Williams, S., Wing, R., \& McCaffery, J. (2016). Partial sleep deprivation impacts impulsive action but not impulsive decision-making. Physiology and Behavior, 164(PtA), 214-219. doi: 10.1016/j.physbeh.2016.06.003

Dickson, S. (2002). How good are elite soccer referees? Just ask the players and coaches! In W. Spink (Ed.), Science and football IV. London: Routledge.

Durmer, J. S., \& Dinges, D. F. (2005). Neurocognitive consequences of sleep deprivation. Seminars in Neurology, 25(1), 117-129. doi: 10.1055/s-2005-867080

Eagles, A., McIellan, C., Hing, W., Carloss, N., \& Lovell, D. (2016). Changes in sleep quantity and efficiency in professional rugby union players during home based training and match-play. The Journal of Sports Medicine and Physical Fitness, 56(5), 565-571. Retrieved from https://www.ncbi.nlm.nih.gov/ pubmed/25369280

Elsworthy, N., Burke, D., Scott, B., Stevens, C., \& Dascombe, B. (2014). Physical and decision-making demands of Australian football umpires during competitive matches. Journal of Strength and Conditioning Research, 28(12), 3502-3507. doi: 10.1519/JSC.0000000000000567

Erlacher,D.,Ehrlenspiel,F., Adegbesan, O., \&El-Din, H.(2011). Sleep habitsin German athletes beforeimportant 
competitions or games. Journal of Sports Sciences, 29(8), 859-866. doi: 10.1080/02640414.2011.565782

Fédération Internationale de Football Association. (2019). Laws of the game: Video Assistant Referee (VAR) protocol (pp. 134-142). Zurich: FIFA.

Frey, D., Badia, P., \& Wright Jr, K. (2004). Inter and intra-individual variability in performance near the circadian nadir during sleep deprivation. Journal of Sleep Research, 13(4), 305-315. doi: 10.1111/j.13652869.2004.00429.x

Gordijn, M., \& Beersma, D. (2007). Circadian control of the sleep-wake cycle. Physiology and Behaviour, 90(2-3), 190-195. doi: 10.1016/j.physbeh.2006.09.010

Gouttebarge, V., Backx, F., Aoki, H., \& Kerkhoffs, G. (2015). Symptoms of common mental disorders in professional football (soccer) across five European countries. Journal of Sports Science and Medicine, 14(4), 811-818. doi: 10.1080/00913847.2017.1248796

Gouttebarge, V., Johnson, U., Rochcongar, P., Rosier, P., \& Kerkhoffs, G. (2017). Symptoms of common mental disorders among professional football referees: A one-season prospective study across Europe. The Physician and Sports Medicine, 45(1), 11-16. doi: 10.1080/00913847.2017.1248796

Hancock, D., Rix-Lievre, G., \& Cote, J. (2015). Citation network analysis of research on sport officials: A lack of interconnectivity. International Review of Sport and Exercise Psychology, 8(1), 95-105. doi: 10.1080/1750984X.2015.1022202

Heinemann, K. (1990). Introduction to sport sociology. Schorndorf: Hofmann.

Helsen, W., \& Bultynck, J.-B. (2004). Physical and perceptual-cognitive demands of top-class refereeing in association football. Journal of Sports Sciences, 22(2), 179-189. doi: 10.1080/02640410310001641502

Hirshkowitz, M., Whiton, K., Albert, S. M., Alessi, C., Bruni, O., Doncarlos, L., Hazen, N., Herman, J., Katz, E., \& Kheirandish-Gozal, L. (2015). National Sleep Foundation's sleep time duration recommendations: Methodology and results summary. Sleep Health, 1(1), 40-43. doi: 10.1016/j.sleh.2014.12.010

Isidori, E., Muller, A., \& Kaya, S. (2012). The referee as educator: Hermeneutical and pedagogical perspectives. Physical Culture and Sport, 56(1), 5-11. doi: 10.2478/v10141-012-0023-2

Jarraya, S., Jarraya, M., Chtourou, H., \& Souissi, N. (2013). Effect of time of day and partial sleep deprivation on the reaction time and the attentional capacities of the handball goalkeeper. Biological Rhythm Research, 45(2), 183-191. doi: 10.1080/09291016.2013.787685

Juliff, L., Halson, S., \& Peiffer, J. (2015). Understanding sleep disturbance in athletes prior to important competitions. Journal of Science and Medicine in Sport, 18(1), 13-18. doi: 10.1016/j.jsams.2014.02.007

Junge, A., \& Feddermann-Demont, N. (2016). Prevalence of depression and anxiety in top-level male and female football players. BMJ Open Sport and Exercise Medicine, 2(1), 1-7. doi: 10.1136/bmjsem-2015-000087

Konishi, K., Kimura, T., Yuhaku, A., Kurihara, T., Fujimoto, M., Hamaoka, T., \& Sanada, K. (2017). Effect of sustained high-intensity exercise on executive function. The Journal of Physical Fitness and Sports Medicine, 6(2), 111-117. doi: 10.7600/jpfsm.6.111

Larkin, P., Mesagno, C., Berry, J., \& Spittle, M. (2018). Exploration of the perceptual-cognitive processes that contribute to in-game decision-making of Australian football umpires. International Journal of Sport and Exercise Psychology, 16(2), 112-124. doi: 10.1080/1612197X.2016.1167760

Lastella, M., Lovell, G., \& Sargent, C. (2014). Athletes' precompetitive sleep behavior and its relationship with subsequent precompetitive mood and performance. European Journal of Sport Science, 14(1), 123-130. doi: 10.1080/17461391.2012.660505

Loewenstein, G., \& Lerner, J. S. (2003). The role of affect in decision making. In R. Davidson, H. Goldsmith, \& K. Scherer (Ed.), Handbook of Affective Science (pp. 619-642). Oxford, Oxford University Press.

MacMahon, C., \& Plessner, H. (2013). The sport official in research and practice. In C. MacMahon, D. Farrow, \& J. Baker, Developing sport expertise - researchers and coaches put theory into practice. London: Routledge.

MacMahon, C., Helsen, W., Starkes, J., \& Weston, M. (2007). Decision-making skills and deliberate practice in elite association football referees. Journal of Sports Sciences, 25(1), 65-78. doi: 10.1080/02640410600718640

MacMahon, C., Mascarhenhas, D., Plessner, H., Pizzera, A., Oudejans, R., \& Raab, M. (2014). Sport officials and officiating: Science and practice. NY: Routledge.

Mallo, J., Frutos, P., Juarez, D., \& Navarro, E. (2012). Effect of positioning on the accuracy of decision making of association football top-class referees and assistant referees during competitive matches. Journal of Sports Sciences, 30(13), 1437-1445. doi: 10.1080/02640414.2012.711485

Martin, B. (1981). Effect of sleep deprivation on tolerance of prolonged exercise. European Journal of Applied Physiology and Occupational Physiology, 47(4), 345-354, doi: 10.1007/BF02332962

Mascarenhas, D., Collins, D., \& Mortimer, P. (2005). Elite refereeing performance: Developing a model for sport science support. The Sport Psychologist, 19(4), 364-379. doi: 10.1123/tsp.19.4.364

Mascarenhas, D., O'hare, D., \& Plessner, H. (2006). The psychological and performance demands of association football refereeing. International Journal of Sport Psychology, 37(2), 99-120. doi: $10.2174 / 1875399$ X0090201000

Morris, G., \& O'Connor, D. (2017). Key attributes of expert NRL referees. Journal of Sports Sciences, 35(9), 852-857. doi: 10.1080/02640414.2016.1194524

Nebes, R., Buysse, D., Halligan, E., Houck, P., \& Monk, T. (2009). Self-reported sleep quality predicts poor cognitive performance in healthy older adults. The Journals of Gerontology, 64B(2), 180-187. doi: 10.1093/ geronb/gbn037

Nevill, A. M., Balmer, N. J., \& Williams, A. M. (2002). The influence of crowd noise and experience upon 
refereeing decision in football. Psychology of Sport and Exercise, 3, 261-272.

Neville, T., Salmon, P., \& Read, G. (2017). Analysis of in-game communication as an indicator of recognition primed decision making in elite Australian rules football umpires. Journal of Cognitive Engineering and Decision Making, 11(1), 81-96. doi: 10.1177/1555343416672557

Page, K., \& Page, L. (2010). Alone against the crowd: Individual differences in referees' ability to cope under pressure. Journal of Economic Psychology, 31(2), 192-199. doi: 10.1016/j.joep.2009.08.007

Pallesen, S., Gundersen, H., Kristoffersen, M., Bjorvatn, B., Thu, E., \& Harris, A. (2017). The effects of sleep deprivation on soccer skills. Perceptual and Motor Skills, 124(4), 812-929. doi: 10.1177/0031512517707412

Peppard, P., Barnet, J., Rasmuson, A., Bliwise, D., \& Hagen, E. (2017). Sleep duration and quality are associated with cognitive performance in midlike and older adulthood. Sleep Medicine, 40(1), 258. doi: 10.1016/j. sleep.2017.11.756

Pina, J., Passos, A., Araújo, D., \& Maynard, M. (2018). Football refereeing: An integrative review. Psychology of Sport and Exercise, 35, 10-26. doi: 10.1016/j.psychsport.2017.10.006

Plessner, H., \& Haar, T. (2006). Sports performance judgments from a social cognitive perspective. Psychology of Sport and Exercise, 7(6), 555-575. doi: 10.1016/j.psychsport.2006.03.007

Rainey, D. (1995). Sources of stress, burnout, and intention to terminate among basketball referees. Journal of Sport Behaviour, 22(4), 578-590. doi: 10.1080/026404199365515

Rainey, D., \& Hardy, L. (1999). Sources of stress, burnout and intention to terminate among rugby union referees. Journal of Sports Sciences, 17, 797-806. doi: 10.1080/026404199365515

Rainey, D., \& Winterich, D. (1995). Magnitude of stress reported by basketball referees. Perceptual and Motor Skills, 81(3), 1241-1242. doi: 10.2466/pms.1995.81.3f.1241

Reilly, T., \& Edwards, B. (2007). Altered sleep-wake cycles and physical performance in athletes. Physiology and Behaviour, 90(2-3), 274-284. doi: 10.1016/j.physbeh.2006.09.017

Rogers, N., Dorrian, J., \& Dinges, D. (2003). Sleep, waking and neurobehavioural performance. Frontiers in Bioscience, 8, 1056-1067. doi: 10.2741/1174

Samuels, C. (2012). Jet lag and travel fatigue: a comprehensive management plan for sport medicine physicians and high-performance support teams. Clinical Journal of Sports Medicine, 22(3), 268-273. doi: 10.1097/ JSM.0b013e31824d2eeb

Scanlan, A., Humphries, B., Tucker, P., \& Dalbo, V. (2014). The influence of physical and cognitive factors on reactive agility performance in men basketball players. Journal of Sports Sciences, 32(4), 367-374. doi: $10.1080 / 02640414.2013 .825730$

Scott, J., McNaughton, L., \& Polman, R. (2006). Effects of sleep deprivation and exercise on cognitive, motor performance and mood. Physiology and Behaviour, 87(2), 396-408. doi: 10.1016/j.physbeh.2005.11.009

Skein, M., Duffield, R., Minett, G., Snape, A., \& Murphy, A. (2013). The effect of overnight sleep deprivation after competitive rugby league matches on postmatch physiological and perceptual recovery. International Journal of Sports Physiology and Performance, 8(5), 556-564. doi: 10.1123/ijspp.8.5.556.

Stewart, B., \& Smith, A. (2000). Australian sport in a postmodern age. The International Journal of the History of Sport, 17(2), 278-304. doi: 10.1080/09523360008714137

Stewart, M., \& Ellery, P. (1996). Amount of psychological stress reported by high school volleyball officials. Perceptual and Motor Skills, 83(1), 337-338. doi: 10.2466/pms.1996.83.1.337

Taylor, A., Daniel, J., Leith, L., \& Burke, R. (1990). Paths from perceived stress to psychological burnout, satisfaction, turnover intentions and actual dropout among soccer officials. Journal of Applied Sport Psychology, 2(1), 84-97. doi: 10.1080/10413209008406422

Thun, E., Bjorvatn, B., Flo, E., Harris, A., \& Pallesen, S. (2015). Sleep, circadian rhythms, and athletic performance. Sleep Medicine Reviews, 23, 1-9. doi: 10.1016/j.smrv.2014.11.003

Watson, N. B. (2015). Joint consensus statement of the American Academy of Sleep Medicine and Sleep Research Society on the recommended amount of sleep for a healthy adult: Methodology and discussion. Journal of Clinical Sleep Medicine, 11(8), 931-52. doi: 10.5665/sleep.4886

Weston, M., Castagna, C., Impellizzeri, F., Bizzini, M., Williams, M., \& Gregson, W. (2012). Science and medicine applied to soccer refereeing. Sports Medicine, 42(7), 615-631. doi: 10.2165/11632360-00000000000000

Williams, A. M., \& Davids, K. (1995). Declarative knowledge in sport: A byproduct of experience or a characteristic of expertise? Journal of Sport and Exercise Psychology, 7(3), 259-275. doi: 10.1123/ jsep.17.3.259

Xing, C., \& Sun, J. (2013). The role of psychological resilience and positive affect in risky decision-making. International Journal of Psychology, 48(5), 935-943. doi: 10.1080/00207594.2012.729840 